\title{
Floating Codes for Joint Information Storage in Write Asymmetric Memories
}

\author{
Anxiao (Andrew) Jiang \\ Computer Science Department \\ Texas A\&M University \\ College Station, TX 77843-3112 \\ ajiang@cs.tamu.edu
}

\author{
Vasken Bohossian \\ Electrical Engineering Department \\ California Institute of Technology \\ Pasadena, CA 91125 \\ vincent@paradise.caltech.edu
}

\author{
Jehoshua Bruck \\ Electrical Engineering Department \\ California Institute of Technology \\ Pasadena, CA 91125 \\ bruck@paradise.caltech.edu
}

\begin{abstract}
Memories whose storage cells transit irreversibly between states have been common since the start of the data storage technology. In recent years, flash memories and other non-volatile memories based on floating-gate cells have become a very important family of such memories. We model them by the Write Asymmetric Memory (WAM), a memory where each cell is in one of $q$ states - state $0,1, \cdots, q-1$ - and can only transit from a lower state to a higher state. Data stored in a WAM can be rewritten by shifting the cells to higher states. Since the state transition is irreversible, the number of times of rewriting is limited. When multiple variables are stored in a WAM, we study codes, which we call floating codes, that maximize the total number of times the variables can be written and rewritten.

In this paper, we present several families of floating codes that either are optimal, or approach optimality as the codes get longer. We also present bounds to the performance of general floating codes. The results show that floating codes can integrate the rewriting capabilities of different variables to a surprisingly high degree.
\end{abstract}

\section{INTRODUCTION}

Memories whose storage cells transit irreversibly between states have been common since the beginning of the data storage technology. Examples include punch cards and digital optical discs, where a cell can change from a 0 -state to a 1state but not vice versa. In recent years, flash memories and some other non-volatile memories based on floating-gate cells have become a very important family of such memories. They have good properties including high data density, fast reading time, physical robustness, etc., and have been widely used in mobile, mass as well as standard storage devices.

We use flash memories as a typical example to explain the basic storage mechanisms based on floating-gate cells. A flash memory consists of floating-gate cells as its basic storage elements. In most products, a cell has two states; but to increase data density, multi-level storage (where a cell has 4 to 256 or even more states) is being developed. For a cell with $q$ states, we denote its states by $0,1, \cdots, q-1$. To write (program) a cell, the hot-electron injection mechanism or the Fowler-Nordheim tunneling mechanism is used to inject electrons into the cell, where the electrons become trapped. The number of trapped electrons in a cell determines the threshold voltage of the cell: the more electrons, the higher the threshold voltage. The number of trapped electrons is chosen to concentrate around $q$ discrete levels, corresponding to the $q$ cell states. The state of a cell can be read by measuring the threshold voltage. Programming and reading cells are fast; however, rewriting data is much more complex. Most of the time, it requires moving cells to lower states for rewriting data, which means to remove electrons from the cells. In flash memories, cells are organized into blocks. A typical block using binary cells stores 64,128 or 256 kilobytes of data. Due to circuit complexity reasons, to rewrite, first the whole block has to be erased (which means to lower all the cells of the block to the 0-state), then all the cells are reprogrammed. This happens even if only one cell really needs to lower its state for the rewriting, and it leads to a writing speed about $10^{5}$ times slower than reading. Therefore, it will be very beneficial to design codes for storing data such that the data can be rewritten many times before the block has to be erased. Reducing the number of block erasing operations is critical not only for reducing the average rewriting time, but also for the flash memory's lifetime. Every erasing reduces the quality of cells, and currently, a flash memory's lifetime is bounded by about $10^{5}$ program-erase cycles. Although technically speaking, a cell can return to a lower state through block erasing, in this paper, we are interested in the writing and rewriting of data between two block erasing operations. In that period, the cells can only go from lower states to higher states.

We model the memories mentioned above using the following Write Asymmetric Memory (WAM) model. A WAM consists of $n$ cells, where each cell has $q$ states: state $0,1, \cdots, q-1$. Such a cell is called a $q$-ary cell. A cell can go from state $i$ to state $j$ if and only if $i<j$.

WAM is a straightforward generalization of the Write Once Memory (WOM) model, firstly proposed by Rivest and Shamir [10], where $q=2$. WAM is also a special case of the Generalized WOM model [3], where the state transition diagram of a cell can be any directed acyclic graph.

Historically, there has been research on WOM codes, where a single variable is stored in a WOM, and the code enables it to be rewritten multiple times [10]. In practice, a memory stores many - let's say $k$ - variables. If we want to apply the codes to a memory, a simple approach is to partition the memory into $k$ parts, where each part stores a variable independently.

This simple approach, however, has a serious limitation. If the rewriting frequencies are very nonuniform for the variables 
- common in many applications - the WAM becomes unusable very soon. For example, say that each storage part allows $t$ times of rewriting of a variable. Once one of the $k$ variables needs rewriting for the $(t+1)$-th time, the WAM can no longer meet the requirement, even if the other $k-1$ variables have not been rewritten yet. Therefore, it will be very beneficial to integrate the rewriting capabilities of the variables, so that the variables can be rewritten many times regardless of what the rewriting sequence is. As we will show in this paper, such an integration is feasible, many times to a surprisingly high degree. We call the codes that achieve it the Floating Codes.

We formally define the problem we study as follows. $k$ variables are stored in a WAM, where each variable takes its value from an alphabet of size $l:\{0,1, \cdots, l-1\}$. The WAM has $n q$-ary cells. Initially, all the cells are in the 0 -state, and all the variables have the default value 0 . Each rewriting updates the value of one variable. We use $\left(v_{1}, v_{2}, \cdots, v_{k}\right)$ - called the variable vector - to denote the values of the $k$ variables, where $v_{i} \in\{0,1, \cdots, l-1\}$. We use $\left(c_{1}, c_{2}, \cdots, c_{n}\right)$ - called the cell state vector - to denote the states of the $n$ cells, where $c_{i} \in\{0,1, \cdots, q-1\}$. A cell state vector $\left(c_{1}, c_{2}, \cdots, c_{n}\right)$ is said to be above another cell state vector $\left(c_{1}^{\prime}, c_{2}^{\prime}, \cdots, c_{n}^{\prime}\right)$ if $c_{i} \geq c_{i}^{\prime}$ for all $i$. When the cells change their states, they can only change to a state vector above the current one.

A floating code has two functions, $\alpha:\{0,1, \cdots, q-1\}^{n} \rightarrow$ $\{0,1, \cdots, l-1\}^{k}$, and $\beta:\{0,1, \cdots, q-1\}^{n} \times\{1,2, \cdots, k\} \times$ $\{0,1, \cdots, l-1\} \rightarrow\{0,1, \cdots, q-1\}^{n}$. Function $\alpha$ maps each cell state vector to a variable vector, which is used to decode (interpret) the stored data. Function $\beta$ shows how to rewrite: given the current cell state vector and the information on which of the $k$ variables is to be updated to which new value, the function $\beta$ outputs the new cell state vector. The new cell state vector should correspond to the new values of the variables.

A floating code allowing times of rewriting is a code that allows the variables to be rewritten at least $t$ times in total, regardless of what the sequence of rewriting is. A fundamental objective of floating codes is to maximize $t$.

In the following, we first present a brief overview of the related work. Then, we present the constructions of several families of floating codes, which either are optimal, or approach optimality as the codes get longer. We also present upper and lower bounds to $t$ for general floating codes.

\section{RELATED WORK}

WOM codes were first studied by Rivest, Shamir [10] et al., where a single variable is stored in a WOM and can be updated multiple times. Capacities of WOMs have been studied [3] [4] [6] [8] [10] [11], and multiple classes of codes have been invented. The majority of those codes are binary, and they include tabular codes [10], linear codes [2] [10], codes constructed using Golay codes [2] or projective geometries [9], etc. Besides WOM, constrained memories also include write efficient memory (WEM), write unidirectional memory (WUM) and write isolated memory (WIM) [8].

There is no work we are aware of that addresses the use of codes for flash memories for increasing the number of (re)writes between two erasing operations, useful for improving writing speed and prolonging the memory lifetime. The use of error-correcting codes for improving data reliability in flash memories has been proposed in some works [1] [5].

\section{AN Optimal CODE For Two BinARY VARIABles}

In this section, we present a floating code for binary variables. That is, $l=2$, so each variable has value 0 or 1 . In flash memories, the 16 bits of a word are usually stored at the same position of 16 parallel blocks. Consequently, a rewriting operation on a word becomes the rewriting of a bit in a block. Therefore, it is important to study the case of $l=2$.

The code we present is for $k=2, l=2$ and arbitrary $n$ and $q$. The code maximizes $t$, the number of rewrites, and is thus optimal. We prove the code's optimality by providing a general upper bound to $t$ for floating codes, which is not limited to the case $k=2, l=2$.

\section{A. Optimal Floating Code for $k=2, l=2$ and Arbitrary $n, q$}

Three examples of the code are shown in Fig. 1, corresponding to $n=1,2$ and 3 , respectively. We comment that $n=1,2$ are, in fact, degenerated cases; it is only when $n=3$ or more that the code reveals the full structure of its construction.

The numbers inside each circle are a cell state vector, while the bold numbers beside the circle are the corresponding variable vector. For example, in Fig. 1(a), the cell state vector $\left(c_{1}\right)=(3)$ corresponds to the variable vector $\left(v_{1}, v_{2}\right)=(0,0)$; in Fig. 1(c), the cell state vector $\left(c_{1}, c_{2}, c_{3}\right)=(1,0,0)$ corresponds to the variable vector $\left(v_{1}, v_{2}\right)=(1,0)$. The arrows leaving a cell state vector shows how the next rewriting should be performed when this cell state vector is the current cell state vector. For example, for the code in Fig. 1(c), if the current cell state vector is $(1,0,0)$ and the new rewriting request is to change the first variable to ' 0 ' (which means to change the variable vector from $(1,0)$ to $(0,0))$, then the cell state vector will become $(1,1,0)$. Similarly, if the sequence of rewriting changes the variable vector as $(0,0) \rightarrow(1,0) \rightarrow$ $(1,1) \rightarrow(0,1) \rightarrow \cdots$ (note that every rewriting changes the value of just one variable), the cell state vector changes as $(0,0,0) \rightarrow(1,0,0) \rightarrow(1,0,1) \rightarrow(1,0,2) \rightarrow \cdots$

We define the cell state vectors of the $i$-th generation to be the cell state vectors reachable after $i$ times of rewriting. In Fig. 1, all the cell state vectors in the same generation are placed at the same horizontal level. For example, in Fig. 1(c), the cell state vectors in the 2 nd generation are $(1,1,0),(1,0,1)$ and $(0,1,1)$. The codes in Fig. 1 are all for arbitrarily large $q$, and they all have periodic patterns; specifically, every code is a repetition of the structure shown in the dotted box labelled by "one period." To see how, notice that the first generation in the dotted box contains two cell state vectors corresponding to two different variable vectors, and so is true for the generation of cell state vectors directly following the dotted box; what's more, the latter two cell state vectors can be obtained from the former two cell state vectors by raising every cell's state by 2. (For example, in Fig. 1(b), the former two cell state vectors are $(1,0)$ and $(0,1)$; when we raise every cell's state by 2 , 

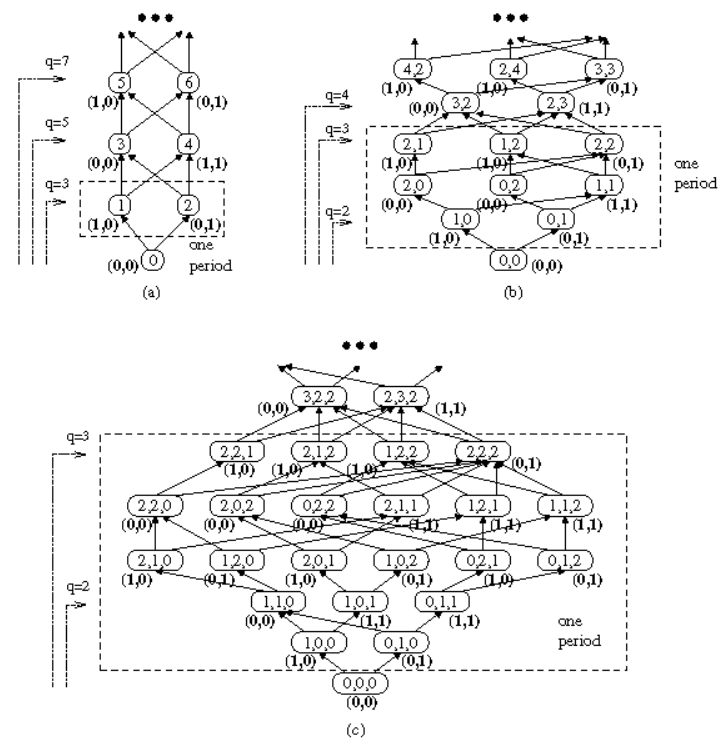

Fig. 1. Three examples of an optimal floating code for $k=2, l=2$ and arbitrary $n$, q. (a) $n=1$. (b) $n=2$. (c) $n=3$.

we get $(3,2)$ and $(2,3)$, the latter two cell state vectors.) The code is built for arbitrarily large $q$ in the following way. A "period" in the code contains $2 n-1$ generations. The second period directly follows - and has the same structure as - the first period, except that: (i) every cell's state is raised by 2, (ii) the pair of variable vectors $(1,0)$ and $(0,0)$ are switched, and the pair of variable vectors $(0,1)$ and $(1,1)$ are also switched. For $i=1,2,3, \cdots$, the $(2 i+1)$-th (resp., $(2 i+2)$-th) period has the same structure as the 1st (resp. 2nd) period except that every cell's state is raised by $4 i$.

If $q$ is finite, it is simple to get the corresponding code: just truncate the above code to the maximum generation, subject to the constraint that every cell's state is at most $q-1$.

We present the formal construction of the code in Fig. 2 to 3. The construction is in fact quite regular and elegant.

It is straightforward to verify the correctness (validity) of the code in Fig. 2 to 3 . The key step is to verify that for every cell state vector, its two outgoing arrows enter two cell state vectors in the next generation that correspond to two different and correct variable vectors $\left(v_{1}, v_{2}\right)$. It is also straightforward to verify the correctness of the following theorem.

Theorem 1: For the code constructed in Fig. 2 and Fig. 3, $t=(n-1)(q-1)+\left\lfloor\frac{q-1}{2}\right\rfloor$.

We see that the floating code integrates the WAM's rewriting capabilities for different variable to a very high degree. Let's call $\sum_{i=1}^{n} c_{i}$ the weight of the cell state vector. Clearly, every rewriting needs to increase that weight by at least 1 . If the $n$ cells are split into two and used independently by the $k=2$ variables, $t$ can never exceed $\frac{n}{2} \cdot(q-1)$. The floating code, however, achieves $t \approx(n-0.5)(q-1)$. The code construction in Fig. 2 to 3 can be easily converted into very efficient encoding (for rewriting) and decoding (for mapping cell state vectors to variable vectors) algorithms. Due to the space limitation, we skip the details.
Floating Code Construction: $k=2, l=2$ and arbitrary $n, q$

1. For $i=1,2, \cdots, n-1$, do:

The $i$-th generation of cell state vectors contains all the $i+1$ elements that satisfy the following properties: (1) Among the first $i+1$ cells, $i$ of them are in state 1 and one of them is in state 0 ; (2) The last $n-(i+1)$ cells are all in state 0.

In the $i$-th generation, if a cell state vector is $(1,1, \cdots, 1,0,0, \cdots, 0)$ (that is, the first $i$ cells are in state 0 , and the last $n-i$ cells are in state 0 ), then it corresponds to the variable vector $\left(v_{1}, v_{2}\right)=(1,0)$ (if $i$ is odd) or $\left(v_{1}, v_{2}\right)=(0,0)$ (if $i$ is even); otherwise, the cell state vector corresponds to the variable vector $\left(v_{1}, v_{2}\right)=(0,1)$ (if $i$ is odd) or $\left(v_{1}, v_{2}\right)=(1,1)$ (if $i$ is even).

Let $a$ denote a cell state vector in the $(i-1)$-th generation. The two outgoing arrows of $a$ are as follows: one arrow goes to the cell state vector in the $i$-th generation where the first $i$ cells are in state 1 and the last $n-i$ cells are in state 0 ; the other arrow goes to the cell state vector of the $i$-th generation that is the same as $a$ except that its $(i+1)$-th cell is in state 1 instead of state 0 .

2. Note that by the above construction, the $(n-1)$-th generation contains $n$ cell state vectors, where each cell state vector has $n-1$ cells in the state 1 and one cell in the state 0 . Let's denote those $n$ cell state vectors by $s_{1}, s_{2}, \cdots, s_{n}$. For $s_{i}(1 \leq i \leq n)$, let's denote the $n-1$ cells in state 1 by $b_{\pi(i, 1)}, b_{\pi(i, 2)}, \cdots, b_{\pi(i, n-1)}$, and denote the cell in state 0 by $b_{\pi(i, n)} .(1 \leq \pi(i, j) \leq n$.)

3. For $i=n, n+1, \cdots, 2 n-3$, do:

The $i$-th generation of cell state vectors contains $n(i-n+2)$ elements, which we partition into $n$ groups. For $j=1,2, \cdots, n$, the $j$-th group contains all the $i-n+2=[i-(n-1)]+1$ elements that satisfy the following properties: among the $[i-(n-1)]+1$ cells $b_{\pi(j, 1)}, b_{\pi(j, 2)}, \cdots, b_{\pi(j, i-(n-1)+1)}, i-(n-1)$ of them are in state 2 and one of them is in state 1 ; the $2 n-i-3$ cells $b_{\pi(j, i-(n-1)+2)}, b_{\pi(j, i-(n-1)+3)}, \cdots, b_{\pi(j, n-1)}$ are all in state 1 ; the cell $b_{\pi(j, n)}$ is in state 0 .

In the $i$-th generation, for a cell state vector in the $j$-th group, if the cells $b_{\pi(j, 1)}, b_{\pi(j, 2)}, \cdots, b_{\pi(j, i-(n-1))}$ are all in state 2 , then it corresponds to the variable vector $\left(v_{1}, v_{2}\right)=(1,0)$ (if $i$ is odd) or $\left(v_{1}, v_{2}\right)=(0,0)$ (if $i$ is even); otherwise, the cell state vector corresponds to the variable vector $\left(v_{1}, v_{2}\right)=(0,1)$ (if $i$ is odd) or $\left(v_{1}, v_{2}\right)=(1,1)$ (if $i$ is even).

Let $a$ denote a cell state vector in the $(i-1)$-th generation and in the $j$-th group. (If $i-1=n-1$, then let $a$ be $s_{j}$.) The two outgoing arrows of $a$ are as follows: one arrow goes to the cell state vector in the $i$-th generation and the $j$-th group where the $i-(n-1)$ cells $b_{\pi(j, 1)}, b_{\pi(j, 2)}, \cdots, b_{\pi(j, i-(n-1))}$ are all in state 2 ; the other arrow goes to the cell state vector in the $i$-th generation and the $j$-th group that is the same as $a$ except that its cell $b_{\pi(j, i-(n-1)+1)}$ is in state 2 instead of state 1 .

4. Note that by the above construction, the $(2 n-3)$-th generation contains $n(n-1)$ cell state vectors, where each vector has $n-2$ cells in state 2, one cell in state 1 , and one cell in state 0 .

5. The $(2 n-2)$-th generation of cell state vectors contains $n+$ $\left(\begin{array}{c}n \\ 2\end{array}\right)$ elements, which we partition into two groups. The first group contains all the $n$ vectors where $n-1$ cells are in state 2 and one cell is in state 0 . The second group contains all the $\left(\begin{array}{c}n \\ 2\end{array}\right)$ vectors where $n-2$ cells are in state 2 and two cells are in state 1 . All the cell state vectors in the first (resp., second) group correspond to the variable vector $\left(v_{1}, v_{2}\right)=(0,0)$ (resp., $(1,1)$ ).

The $(2 n-1)$-th generation of cell state vectors contains $n+$ 1 elements, which we partition into two groups. The first group contains all the $n$ cell state vectors where $n-1$ cells are in state 2 and one cell is in state 1 ; the second group contains one cell state vector where all the $n$ cells are in state 2 . The cell state vectors in the first (resp. second) group correspond to the variable vector $\left(v_{1}, v_{2}\right)=(1,0)$ (resp. $\left.(0,1)\right)$.

Let $a$ denote a cell state vector in the $(2 n-3)$-th (resp., $(2 n-$ 2 )-th) generation. The two outgoing arrows of $a$ enter two cell state vectors of the $(2 n-2)$-th (resp., $(2 n-1)$-th) generation, respectively in the first group and in the second group, both of which are above a. (To be continued in Fig. 3.)

Fig. 2. 


\section{Floating Code Construction: Continuation of Fig. 2}

6. The above $2 n-1$ generations of cell state vectors form the first period of the code. Repeat the period's structure to get the 2nd, 3rd, . . periods (as described before in this paper). Just remember that for the $i$-th period, if $i$ is even, then switch the variable vector $(0,0)$ with $(1,0)$, and switch the variable vector $(1,1)$ with $(0,1)$. If $q$ is finite, truncate the code to the maximum generation subject to the constraint that all the cells' states are at most $q-1$

Fig. 3.

\section{B. A General and Tight Upper Bound to $t$}

We now present a general upper bound to $t$, which holds for any $k, l, n$ and $q$. The bound can show that the code in Fig. 2 and Fig. 3 is optimal.

Theorem 2: For any floating code, if $n \geq k(l-1)-1$, then $t \leq[n-k(l-1)+1] \cdot(q-1)+\left\lfloor\frac{[k(\bar{l}-1)-1] \cdot(q-1)}{2}\right\rfloor$; if $n<k(l-1)-1$, then $t \leq\left\lfloor\frac{n(q-1)}{2}\right\rfloor$.

Proof: Due to the space limitation, we present only the sketch of the proof here. Please refer to [7] for the details. The idea is to show that for any floating code, when $n \geq k(l-1)-$ 1 , there is a sequence of at most $[n-k(l-1)+1] \cdot(q-1)+$ $\left\lfloor\frac{[k(l-1)-1] \cdot(q-1)}{2}\right\rfloor$ rewriting operations after which no more rewriting can be performed. The case $n<k(l-1)-1$ can be analyzed similarly. We find such a "bad" sequence of rewriting operations using the following method. For $i=0,1,2 \ldots$, after the $i$-th rewriting operation, we use a set $S_{i}$ to remember the $k(l-1)-1$ cells whose states are the lowest. The $i$-th rewriting operation is selected in this way: if all the choices for the rewriting increases the weight of the cell state vector by only one, we select the $i$-th rewriting operation to be the one that raises the state of a cell outside $S_{i-1}$; otherwise, we select the $i$-th rewriting operation to be the one that increases the weight of the cell state vector by at least two.

Let $a_{1}, a_{2}, \cdots, a_{n}$ denote the $n$ cells, and let $c_{j}^{i}$ denote the state of the $j$-th cell after the $i$-th rewriting operation. $(0 \leq$ $c_{j}^{i} \leq q-1$.) Let $P_{i}=\sum_{a_{j} \in S_{i}}\left(q-1-c_{j}^{i}\right)$, and let $Q_{i}=$ $\sum_{a_{j} \notin S_{i}}\left(q-1-c_{j}^{i}\right)$. Let $t_{0}$ denote the number of rewriting operations in our rewriting sequence. With a careful inductive analysis, we show that for all $i, t_{0}-i \leq Q_{i}+\left\lfloor\frac{P_{i}}{2}\right\rfloor$. By replacing $i$ with 0 , we obtain the final conclusion.

By plugging $k=2$ and $l=2$ into Theorem 2, and comparing it with Theorem 1, we get the following conclusion.

Theorem 3: The floating code presented in Fig. 2 and Fig. 3 is optimal, namely, it maximizes the number of rewrites $t$.

The above observation also shows that in some cases, the upper bound presented in Theorem 2 is tight.

\section{NeARly Optimal LinEAR CODES}

In this section, we present two linear codes of similar structures for binary variables. Both codes have $\lim _{n \rightarrow \infty} t=$ $(q-1) n+o(n)$. Since all floating codes have $t \leq(q-1) n$, the two codes approach optimality as $n \rightarrow \infty$.

In both codes, every cell essentially corresponds to an integer, and a linear combination of those integers form the numerical representation of the $k$ variables. We borrow the idea from the WOM codes proposed by Fiat and Shamir in [3].
Those WOM codes are for updating a single variable in a binary WOM. The floating codes we present are, respectively, for rewriting two or three variables in $q$-ary WAMs.

We define a function odd $(x)$ as follows: for any nonnegative integer $x$, if $x$ is odd, odd $(x)=1$; otherwise, $\operatorname{odd}(x)=0$. Let $\left(a_{1}^{x_{1}} a_{2}^{x_{2}} \cdots a_{h}^{x_{h}}\right)$ denote a string that consists of $x_{1}$ consecutive $a_{1}$ 's, followed by $x_{2}$ consecutive $a_{2}$ 's, $\cdots$, ended with $x_{h}$ consecutive $a_{h}$ 's. For example, $\left(1^{2} 0^{1} 1^{1} 0^{3}\right)$ is $(1,1,0,1,0,0,0)$.

Below are the constructions of the two floating codes.

- Code Construction $I: k=2, l=2, n \geq 3$, arbitrary $q$ In this code, a valid cell state vector $\left(c_{1}, c_{2}, \cdots, c_{n}\right)$ always satisfies the following two constraints: (1) $\forall i, j$, $\left|c_{i}-c_{j}\right| \leq 1 ;(2)\left(c_{1}, c_{2}, \cdots, c_{n}\right)=\left((a+1)^{x_{1}} a^{x_{2}}(a+\right.$ $1)^{x_{3}}$ ) for some $a, x_{1}, x_{2}, x_{3}$ where $0 \leq a<q-1, x_{1}+$ $x_{2}+x_{3}=n, x_{2} \geq 1$. (For example, when $n=5, q=3$, $(1,1,1,0,1)=\left(1^{3} 0^{1} 1^{1}\right)$ and $(1,1,1,1,2)=\left(2^{0} 1^{4} 2^{1}\right)$ are both valid cell state vectors.)

A cell state vector $\left((a+1)^{x_{1}} a^{x_{2}}(a+1)^{x_{3}}\right)$ corresponds to the variable vector $\left(v_{1}, v_{2}\right)$ in the following way: $v_{1}=$ $\operatorname{odd}\left(x_{1}\right), v_{2}=\operatorname{odd}\left(x_{3}\right)$.

The rewriting operation is as follows. When the rewriting changes the value of variable $v_{1}$ (resp., $v_{2}$ ), we usually increase $x_{1}$ (resp., $x_{3}$ ) by 1 and decrease $x_{2}$ by 1 . The exception happens when $x_{2}=1$; in that case, we first raise all the cells to the state $a+1$ (which makes $x_{1}=$ $x_{3}=0$ and $x_{2}=n$ ), then increase $x_{1}$ or $x_{3}$ (or both) based on necessity.

For example, assume that $n=4, q=3$ and the rewriting operations change the variable vector $\left(v_{1}, v_{2}\right)$ as follows: $(0,0) \rightarrow(0,1) \rightarrow(0,0) \rightarrow(1,0) \rightarrow(1,1)$. Then, the cell state vector changes as follows: $(0,0,0,0) \rightarrow$ $(0,0,0,1) \rightarrow(0,0,1,1) \rightarrow(1,0,1,1) \rightarrow(2,1,1,2)$.

Theorem 4: When $n$ is odd, the floating code in Code Construction I has $t=(n-1)(q-1)$; if $n$ is even, it has $t=(n-2)(q-1)+1$.

We skip the proof for theorem 4 due to the space limitation. Interested readers please see [7]. By theorems 2 and 4, we see that when $q=2$, the above code is strictly optimal.

- Code Construction II: $k=3, l=2, n \geq 5$, arbitrary $q$ In this code, a valid cell state vector $\left(c_{1}, c_{2}, \cdots, c_{n}\right)$ always satisfies the following two constraints: (1) $\forall i, j$, $\left|c_{i}-c_{j}\right| \leq 1 ;(2)$ the cell state vector is either in the form $\left((a+1)^{x_{1}} a^{x_{2}}(a+1)^{x_{3}} a^{x_{4}}(a+1)^{x_{5}}\right)$, where $\sum_{i=1}^{5} x_{i}=$ $n, x_{2} \geq 1, x_{4} \geq 1$ (which we call form $I$ ), or in the form $\left((a+1)^{x_{1}} a^{x_{2}}(a+1)^{x_{5}}\right)$, where $x_{1}+x_{2}+x_{5}=n, x_{2} \geq 1$ (which we call form $I I)$.

A cell state vector corresponds to the variable vector $\left(v_{1}, v_{2}, v_{3}\right)$ in the following way: if the cell state vector is in form $I$, then $v_{1}=\operatorname{odd}\left(x_{1}\right), v_{2}=\operatorname{odd}\left(x_{3}\right)$, $v_{3}=o d d\left(x_{5}\right)$; if the cell state vector is in form II, then $v_{1}=\operatorname{odd}\left(x_{1}\right), v_{2}=0, v_{3}=\operatorname{odd}\left(x_{5}\right)$.

The rewriting operation is as follows. When the rewriting changes the value of variable $v_{1}$ (resp., $v_{3}$ ), we usually increase $x_{1}$ (resp., $x_{5}$ ) by 1 and decrease $x_{2}$ (resp., $x_{4}$ or 
$x_{2}$, depending on if the cell state vector is in form $I$ or form $I I$ ) by 1 . When the rewriting changes the value of variable $v_{2}$, we either increase $x_{3}$ by 1 and decrease $x_{2}$ or $x_{4}$ by 1 (when the cell state vector is in form $I$ ), or change the cell in the middle of the sequence of $a$ 's from state $a$ to state $a+1$ (when the cell state vector is in form II). If $x_{2}$ or $x_{4}$ becomes zero due to the above operation, the cell state vector is reevaluated, and the operation described above is carried out again based on the values of the variables. If the above operation cannot be carried out any more when the cells remain in the current two states - state $a$ and state $a+1$ - then we start to use the two states $a+1$ and $a+2$, in the same way as we have used the two states $a$ and $a+1$ above.

The following examples show how the code works. Assume that $n=10, q=3$. (1) If the cell state vector is $\left(1^{1} 0^{9} 1^{0}\right)$, then $\left(v_{1}, v_{2}, v_{3}\right)=(1,0,0)$; if the next two rewriting operations change $\left(v_{1}, v_{2}, v_{3}\right)$ to $(0,0,0)$ and then to $(0,1,0)$, the cell state vector changes to $\left(1^{2} 0^{8} 1^{0}\right)$, and then to $\left(1^{2} 0^{3} 1^{1} 0^{4} 1^{0}\right)$. (2) If the cell state vector is $\left(1^{3} 0^{1} 1^{1} 0^{4} 1^{1}\right)$, then $\left(v_{1}, v_{2}, v_{3}\right)=(1,1,1)$; if the next two rewriting operations change $\left(v_{1}, v_{2}, v_{3}\right)$ to $(0,1,1)$ and then to $(0,1,0)$, the cell state vector changes to $\left(1^{6} 0^{1} 1^{1} 0^{1} 1^{1}\right)$, and then to $\left(2^{0} 1^{4} 2^{1} 1^{5} 2^{0}\right)$.

Theorem 5: The floating code in Code Construction II has $t \geq\left(n-6-2 \log _{2} n\right)(q-1)+2$.

Proof: We present the sketch of the proof here because of the space limitation. Please see [7] for details. The main idea is that every rewriting increases the weight of the cell state vector only by one except in the following two cases: (1) The rewriting makes $x_{2}$ or $x_{4}$ become zero, in which case the weight of the cell state vector can be increased by at most 3; (2) The rewriting causes the cells to stop using the current pair of states - say $a$ and $a+1$ - and start using the next pair of states $a+1$ and $a+2$, in which case the weight of the cell state vector is increased by at most 7 . The first case can be shown to occur no more than $(q-1) \log _{2} n$ times, while the second case happens at most $q-1$ times. That leads to the final conclusion. For the detailed proof, please see [7].

\section{Bounds For FloAting CODES}

A general upper bound to $t$ was shown in Theorem 2. It was also shown that when $k=2, l=2$, the bound is exact. For large $k$ or $l$, the following theorem can give a better bound.

Theorem 6: Let $w$ be the smallest positive integer such that $\left(\begin{array}{c}w+n \\ n\end{array}\right) \geq l^{k}$. Then, $t \leq\left\lceil\frac{(q-1) n}{w}\right\rceil k$.

Let $w^{\prime}$ be the smallest positive integer such that $\left(w^{\prime}+n\right)>$ $l^{k}$. Then, when $k \geq 2, t \leq\left\lceil\frac{(q-1) n}{w^{\prime}}\right\rceil k$.

Proof: First, consider the general case $k \geq 1$. Define $S$ as $S=\left\{\left(a_{1}, a_{2}, \cdots, a_{n}\right) \mid \sum_{i=1}^{n} a_{i} \leq w, a_{1}, a_{2}, \cdots, a_{n}\right.$ are nonnegative integers $\}$, and let $w$ be the smallest integer such that $|S| \geq l^{k}$. Define $S^{\prime}$ as $S^{\prime}=\left\{\left(d_{1}, d_{2}, \cdots, d_{n}\right) \mid \sum_{i=1}^{n} d_{i} \leq w+\right.$ $n, d_{1}, d_{2}, \cdots, d_{n}$ are positive integers $\}$. By letting $d_{i}=a_{i}+1$ for $i=1,2, \cdots, n$, we see that there is a one-to-one mapping between $S$ and $S^{\prime}$. So $|S|=\left|S^{\prime}\right|$. An element $\left(d_{1}, d_{2}, \cdots, d_{n}\right)$ belongs to $S^{\prime}$ if and only if it is a solution to the following problem: partition a path of $w+n$ vertices into $n$ or more sub-paths such that for $i=1,2, \cdots, n$, the $i$-th sub-path has $d_{i}>0$ vertices. Therefore, $\left|S^{\prime}\right|=\left(\begin{array}{c}w+n \\ n\end{array}\right)$. So $w$ is also the smallest positive integer such that $\left(\begin{array}{c}w+n \\ n\end{array}\right) \geq l^{k}$.

$k$ consecutive rewriting operations can make the variables change to or go through any of the $l^{k}$ possible values. If we see $a_{i}$ (for $i=1,2, \cdots, n$ ) as the increase in $c_{i}$ - the state of the $i$-th cell - and consider the way $S$ and $w$ are defined, we see that whatever the current cell state vector is, there exist $k$ consecutive rewriting operations that increases the weight of the cell state vector $\sum_{i=1}^{n} c_{i}$ by at least $w$. Now consider the first batch of such $k$ rewriting operations, the second batch, and so on. Since the maximum weight of the cell state vector is $(q-1) n$, we get $t \leq\left\lceil\frac{(q-1) n}{w}\right\rceil k$.

The proof for the slightly more restrictive case $k \geq 2$ is very similar. Please see [7] for its details.

When $k$ or $l$ is sufficiently large, theorem 6 gives an upper bound to $t$ that is roughly $\frac{(q-1) n k}{(n !)^{\frac{1}{n}} l^{\frac{k}{n}}}$. Now we present an elementary lower bound as well as an exact evaluation for $t$. Due to the space limitation, we skip their proofs. Please refer to [7] for the detailed proofs.

Theorem 7: There exist floating codes where $t \geq\left\lfloor\frac{n}{k}\right\rfloor$. $\left\lfloor\frac{q-2}{l-1}\right\rfloor$.

Theorem 8: When $k, l, q$ are fixed and $n \rightarrow \infty$, there exist floating codes where $t=(q-1) n+o(n)$.

Theorem 8 shows that when $n \rightarrow \infty$, floating codes can integrate the WAM's rewriting capabilities for different variables nearly perfectly. Such a nearly perfect integration also occurs to all the codes presented in this paper.

\section{ACKNOWLEDGMENT}

This work was supported in part by the Lee Center for Advanced Networking at the California Institute of Technology.

\section{REFERENCES}

[1] P. Cappelletti, C. Golla, P. Olivo and E. Zanoni (Ed.), Flash memories, Kluwer Academic Publishers, 1st Edition, 1999.

[2] G. D. Cohen, P. Godlewski and F. Merkx, "Linear binary code for writeonce memories," IEEE Trans. Inform. Theory, vol. IT-32, pp. 697-700, Sept. 1986.

[3] A. Fiat and A. Shamir, "Generalized 'write-once' memories," IEEE Trans. Inform. Theory, vol. IT-30, pp. 470-480, May 1984.

[4] F. Fu and A. J. Han Vinck, "On the capacity of generalized write-once memory with state transitions described by an arbitrary directed acyclic graph," IEEE Trans. Inform. Theory, vol. 45, no. 1, pp. 308-313, 1999.

[5] S. Gregori, A. Cabrini, O. Khouri and G. Torelli, "On-chip error correcting techniques for new-generation flash memories," Proceedings of The IEEE, vol. 91, no. 4, April 2003.

[6] C. Heegard, "On the capacity of permanent memory," IEEE Trans. Inform. Theory, vol. IT-31, pp. 34-42, Jan. 1985.

[7] A. Jiang, V. Bohossian and J. Bruck, "Floating codes for joint information storage in write asymmetric memories," electronic technical report, http://faculty.cs.tamu.edu/ajiang/Floatingcodes.pdf, 2007.

[8] A. V. Kuznetsov and A. J. H. Vinck, "On the general defective channel with informed encoder and capacities of some constrained memories," IEEE Trans. Inform. Theory, vol. 40, no. 6, pp. 1866-1871, Nov. 1994.

[9] F. Merkx, "WOMcodes constructed with projective geometries," Traitement du Signal, vol. 1, no. 2-2, pp. 227-231, 1984.

[10] R. L. Rivest and A. Shamir, "How to reuse a 'write-once' memory," Information and Control, vol. 55, pp. 1-19, 1982 .

[11] J. K. Wolf, A. D. Wyner, J. Ziv and J. Korner, "Coding for a write-once memory," AT\&T Bell Labs. Tech. J., vol. 63, no. 6, pp. 1089-1112, 1984. 